\title{
A view of covalent and ionic bonding from Maximum Probability Domains
}

\author{
M. Menéndez ${ }^{\mathrm{a}, \mathrm{b}}$, A. Martín Pendás ${ }^{\mathrm{a}}$, B. Braïda ${ }^{\mathrm{b}, \mathrm{c}, *}, \mathrm{~A}$. Savin ${ }^{\mathrm{b}, \mathrm{c}}$ \\ a Departamento de Química Física y Analítica, Facultad de Química, Universidad de Oviedo, 33006 Oviedo, Spain \\ ${ }^{\mathrm{b}}$ Sorbonne Universités, UPMC Univ. Paris 06, UMR 7616, LCT, F-75005 Paris, France \\ ${ }^{\mathrm{c}}$ CNRS, UMR 7616, LCT, F-75005 Paris, France
}

Keywords:

Topological analysis

Chemical bonding

Real space technique

\begin{abstract}
A B S T R A C T
Connecting the accurate Quantum Mechanics to the chemical view is the first of foremost purposes of interpretative methods in general, and topological analysis in particular. In this field of methods, the Maximum Probability Domains (MPD) analysis, is conceptually appealing but has not been extensively applied yet. In this study we provide the general vision coming out from MPD on the two main family of bonds: polar-covalent and ionic bonds. An interesting picture arises concerning the MPD solution associated to covalent bonds, displaying a prolate shape that extends preferentially in the orthogonal direction to the bond axis, and not along it. The polarity of the bond only affects marginally the domain shape, though further probability analysis seems to allow quantifying it. Concerning the ionic bond, a resonating picture emerges, which is compatible, and refines, the usual electrostatic vision of two oppositely-charged atoms in interaction.
\end{abstract}

\section{Introduction}

In his 1916 seminal paper The atom and the molecule, Lewis [1] introduced his original model of the cubical atom and electron pairing to rationalize bonding in molecules. This model, supplemented by Langmuir's octet rule [2] can be considered as the foundation of the modern chemical alphabet, and as such it determines the way chemists see and think the concept of chemical bond. Later, Linus Pauling, in the first of his famous series of articles on the nature of the chemical bond [3], connected the recently developed quantum theory with the Lewis model, and presented the electron-pair bond (the covalent bond in modern terms) and the ionic bond as the two extreme cases of bonding mechanisms which can lead to a two electron chemical bond between two atoms. As depicted in Scheme 1a, in Pauling's electron-pair bond the stabilization comes from spin-exchange resonance energy of the two singlet-coupled electrons, which is a purely quantum effect, whereas for the ionic bond (Scheme 1b) it is the classical electrostatic interaction between the two ions that drives the stabilization. Much more recently, a third distinct type of bonding mechanism has been identified, the "charge shift bonding" mechanism, where the bonding stabilization does not come

* Corresponding author at: Sorbonne Universités, UPMC Univ. Paris 06, UMR 7616, LCT, F-75005 Paris, France.

E-mail address: benoit.braida@upmc.fr (B. Braïda). neither from the spin-pairing nor from Coulomb interaction between ions, but from the covalent-ionic resonance energy [4]. Thinking in terms of these three extreme bonding concepts is useful, as the different bonding types lead to different experimental manifestations, such as strong dipolar moments or heterolytic cleavage in polar solvents for ionic bonds.

Since then, chemists minds have been shaped by Lewis' localized vision and Pauling's classification in terms of covalent and ionic bonds. This vision has influenced the way chemists apprehend how molecules interact and react together, and hence chemical intuition. It has thus been of major importance to develop interpretative methods, which, on one hand, enable a connection between the results of accurate quantum mechanical calculations and the expectations of chemists, and, on the other hand, enhance the chemists' vision in small brush strokes with new information extracted from quantum calculated densities or wave functions. One such method is the Valence Bond (VB) theory, which after being almost abandoned seems to get a new creative twist [5-7]. However, it suffers from two important limitations. The first limitation is technical in nature, as the range of systems and problems which could be tackled with modern VB computational methods is still quite restricted, and the know-how (both theoretical and technical) needed to apply such methods is significant, even if impressive progress has been made lately in both aspects [8]. The second limitation is more fundamental: VB theory, such as the popular and in former times rival Molecular Orbital (MO) theory, operates in 
orbital space, and thus delivers images which are valid in this space, but are usually implicitly assumed to depict what is happening in real space. An interpretative method would be most useful when ideally fulfilling the following requirements: (i) it can retrieve key chemical concepts, and particularly the concept of chemical bonding, from accurate quantum calculations; (ii) it delivers chemical images in real space; (iii) it is kept as simple as possible: the definition and physical meaning of the interpretative quantities computed with the method can be readily understood by most experimental chemists; (iv) it can generate explanatory models which rationalize a whole bunch of data, and allows predictions to be made. The Atoms in Molecules (AIM) [9] and Electron Localization Function (ELF) $[10,11]$ methods are such successful real space density based interpretative methods. More recently, the $\mathrm{NCI}$ method has been formulated which allows semi-quantitative information about intermolecular interactions $[12,13]$.

A few years ago, a new method to analyse the distribution of electrons in regions of space was proposed [14]. The basic quantity which is computed and analyzed is defined in Eq. (2) below, and could simply be defined as the probability to find $v$ and only $v$ electrons in the region of space $\Omega$, extracted from a given wave function, $\Psi$, describing an $N$-electron system.

$$
\begin{aligned}
p_{v}(\Omega)= & \left(\begin{array}{c}
N \\
v
\end{array}\right) \int_{\Omega} d \mathbf{r}_{1} d \mathbf{r}_{2} \ldots d \mathbf{r}_{v} \\
& \times \int_{\bar{\Omega}} d \mathbf{r}_{v+1} d \mathbf{r}_{v+2} \ldots d \mathbf{r}_{N}\left|\Psi\left(\mathbf{r}_{1}, \mathbf{r}_{2}, \ldots, \mathbf{r}_{\mathbf{N}}\right)\right|^{2}
\end{aligned}
$$

where the physical space, $R^{3}$, is divided such that $\Omega$ and $\bar{\Omega}$ exhaust it, i.e. $R^{3}=\Omega \cup \bar{\Omega}$. This probability analysis has first been carried out on ELF and AIM basins [15], and later extended to the full Electron Distribution Function (EDF) analyzed on a whole AIM spacepartitioning [15-18]. It is more logical though, instead of using regions of space generated by other - and quite different - interpretative methods, to search for the regions of space maximizing the probability to find a given number of electrons $v$ (Eq. (3)). Such regions are called Maximum Probability Domains (MPDs), are denoted with $\Omega_{v}$, and stand for the domains that maximize the probability to find $v$ and only $v$ electron inside it.

$\Omega_{v}=\max _{\Omega} p_{v}(\Omega)$

An interpretative method built around these MPDs may fulfil the four requirements listed above. First, the domains maximizing the probability to find 2 (and only 2) electrons, namely $\Omega_{2}$, may be directly assimilated to Lewis' electron pairs, extracted from the wavefunction $\Psi$. In particular, MPDs can provide direct images in real space of chemical bonds. As it will be seen, the concept of resonating situations, fundamentally probabilistic in nature, also comes out naturally. Besides, the fundamental quantities of the method, i.e. the probability function and the MPDs are at the same time mathematically very simple, easy to formulate, and conceptually simple to understand. Given the huge amount of know-how that practicing chemists have gathered over the last century around predicting chemical behavior from Lewis' concepts, MPDs may become a rigorous entry point to standard chemical concepts from wave functions. After some early applications using a toy program [19] as well as some molecular Quantum Monte Carlo calculations [20], lately the focus has been more on analysing the

$$
\begin{aligned}
& \mathrm{A} \cdot+\cdot \mathrm{B} \Longrightarrow \mathrm{A}: \mathrm{B}=\mathrm{A} \cdot \stackrel{\downarrow}{\natural} \mathrm{B} \leftrightarrow \mathrm{A}^{\downarrow} \cdot \stackrel{\uparrow}{ } \cdot \mathrm{B} \\
& \mathrm{A} \cdot+\mathrm{B} \Longrightarrow \mathrm{A}^{\oplus} \stackrel{\ominus}{:} \mathrm{B}
\end{aligned}
$$

Scheme 1. Two extreme cases of bonding mechanisms leading to a two electron bond. basic properties of MPDs [21,22] and on developing algorithms and programs able to efficiently optimize these domains for molecules and solids, which is still an on-going effort. Some fundamental application on basic solid-state systems has also been carried out lately [23]. The purpose of the present work is to display and analyse the standard pictures coming from MPD analysis applied to some molecules representative of the two main families of chemical bonds, namely covalent and ionic bonds. It will be shown that both informative and quite different views of these bonding types come out from MPDs.

\section{Technical details}

We have considered several systems to analyze the images provided by the MPD method. These include closed-shell atoms and molecules with a ground state described by a single-determinant wave function. The chosen systems are the neon atom and the anions of the halogens. For molecules, hydrogen halides (HX) and lithium halides ( $\mathrm{LiX})(\mathrm{X}=\mathrm{F}, \mathrm{Cl}, \mathrm{Br}, \mathrm{I})$ were studied as also the dinitrogen, ethane in its staggered conformation and the trifluorimethyltrimethylsilane (TFMTMS) $\mathrm{F}_{3} \mathrm{SiMe}_{3}$ molecules. All the electronic structure calculations have been performed with the Gaussian code [24] making use of the Density Functional Theory (DFT) method with the hybrid B3LYP functional. As later explained in Section 3, the use of pseudopotentials was preferred to avoid different technical issues. To that end, the VTZ Burkatzki basis sets and pseudopotentials [25] were employed for all atoms in all molecules, except for the hydrogen ones, where an improved pseudopotential developed by Petruzielo et al. [26] was used. For the neon atom, the cc-pVTZ correlation consistent basis set developed by Dunning and coworkers [27] has been also employed.

Focusing on the Maximum Probability Domains calculations, these have been done by applying the MPD code developed by $\mathrm{O}$. Mafra [28]. The program makes use of a grid of small cubes as unit elements. To represent a spatial domain $\Omega$, a set of such cubes is needed. The optimization procedure of the $\Omega$ domain is performed on a precomputed parallelepipedic spatial grid enclosing the molecule. It starts by guessing an initial region formed by the union of a collection of grid cubes located in the part of space of interest, or by using the basins obtained by the ELF method making use of the TopMod program [29]. Two different optimization algorithms are available. In one of them, grid cubes are randomly added or deleted, a step being accepted when the desired probability increases. Since obtaining a MPD is a problem in shape optimization that relies on the concept of shape derivative, the second algorithm implemented so far makes uses of these derivatives in order to indicate where cubes should be added or deleted to increase the probability. This improved algorithm has already been presented in more detail in reference [35], and has been the one which has been employed in all our calculations.

In order to guarantee the accuracy of our results, several tests have been carried out to gauge the importance of the computational parameters determining the fineness of the grid. These are the size of the search region and the number of intervals in which it is divided. Both define the physical dimensions of the small cubes that comprise the grid. After several tests, we have concluded that a good compromise between computer time and accuracy is obtained if the edge of the cube elements of the grid (the stepsize) is set around $0.15 \mathrm{bohr}$ whenever pseudopotentials are used. With these considerations, we are confident that all the quantities computed (probabilities, average electronic populations inside domains, etc.) are accurate enough. For instance, the probabilities presented in this paper are accurate to about three digits. Thinner grids have shown to increase computer time significantly with no clear quality improvement. 
Another way to check the quality of the grid is to extend it over the whole molecule. The probability to find in it all $\mathrm{N}$ electrons should be close to one, while any other probability close to zero. For obtaining a given MPD, the grid should only be able to cover it. However, taking into consideration the smallness of the molecules considered in this paper, we have always chosen a for all molecules and all MPDs a grid extending over the entire molecular space. All the grids used in this work are cubic, with the center of mass of each molecule at the cube's center. Edges equal to 16 a.u. (thus corresponding to 110 subdivisions and a stepsize of 0.145 a.u.) were used in the hydrogen halides, LiF, the monoatomic anions of the halogens, ethane, dinitrogen and the Ne atom systems. A larger 20 a.u. edge with 130 subdivisions was used in $\mathrm{LiCl}$, $\mathrm{LiBr}$, Lil and the TFMTMS compounds. These stepsizes have been chosen so that the full grids contain the total number of electrons up to 0.01. As discussed below, all-electron calculations need thinner grids to properly optimize both core and valence MPDs displaying boundaries with the cores. To that end, a full grid with 16 a.u. edge and 300 subdivisions was used in the case of all-electron calculations presented for the neon atom.

An important remark regarding domain optimization needs to be made. A MPD may either enclose a finite volume or extend towards infinity along some directions. In the latter cases, much of the volume of such MPDs will correspond to very small density regions that have little significance and preclude an appealing visualization of their shape. To that end, we have added a $\varepsilon V$ term to the $p_{v}$ target functional ( $\varepsilon$ being small and negative). This term reduces the volume of the final MPD as if it were compressed by some external pressure. The final constrained MPDs have finite volumes, and display target probabilities which are marginally different from the unconstrained ones, with average electron populations differing in less than 0.01 electrons. We have made wide use of the $\varepsilon V$ constraint in our calculations with $\varepsilon=-10^{-7} \mathrm{au}$.

The XMVB [30] program coupled to the GAMESS [31] package has been used to perform the Valence Bond calculations. The Breathing Orbital Valence Bond (BOVB) method was used, a variational ab initio $\mathrm{VB}$ method which allows both the inclusion of static correlation through the multi-structure expansion, and the inclusion of differential dynamical correlation through the use of different orbitals for different structures [32]. This method has several levels of sophistication, and the so-called D-BOVB (Delocalized Breathing Orbitals Valence Bond) method has been selected for this work. The structure weights are then computed from the optimized structure coefficients and structure overlap matrices using the Coulson-Chirgwin formula [33]. Calculations has been carried out on the same optimized geometries and using the same basis set as for the corresponding Maximum Probability Domains. Notice, however, that our MPDs were always obtained through the single determinant pseudo wave functions provided by DFT calculations.

All pictures have been obtained with the Jmol [34] visualization program.

\section{Results and discussion}

\subsection{Atoms}

Before moving to the molecular case, it will be useful to present the Maximum Probability Domains that come out for a single valence full atom. This will serve as a reference for the following, in particular for the case of ionic systems, and allows to present some typical properties of MPDs on the simplest possible "chemical" case. It should be noted, as explained before [14,19,21], that in general multiple MPD solutions exists. Fig. 1 displays the typical $\Omega_{2}$ domains that can be obtained for the neon atom, i.e. the domains
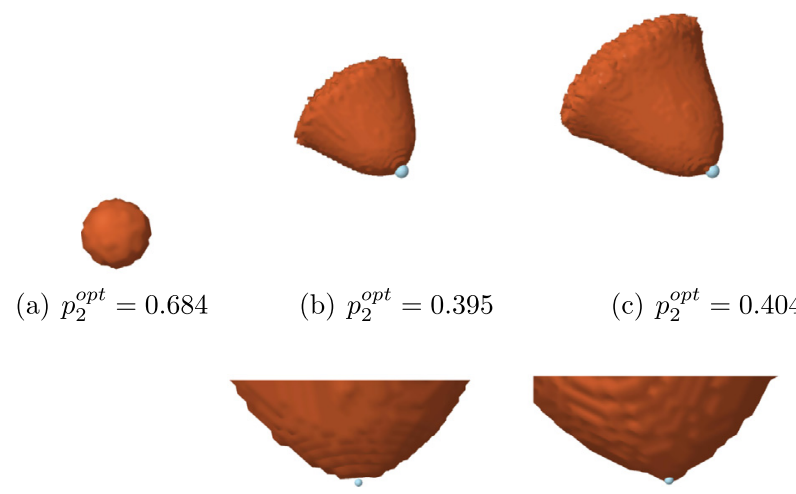

Fig. 1. Different $\Omega_{2}$ MPD's obtained for the neon atom for all-electron (left and middle) and pseudopotential calculations (right). The domain on the left (on a different scale with respect to the middle and the left ones) corresponds to the core pair, and those on the middle and right pictures to one of the valence solutions. There are an infinite number of similar solutions in the latter case, obtained by an arbitrary rotation. The values given correspond to the maximized probabilities. For the (b and c) domains, a close-up image of the domains around the nuclear region is also shown (bottom images) in order to appreciate how the nucleus is included in the pseudopotential calculation, leading to the sharpening of the MPD shape commented on the text.

maximizing $p_{2}$, the probability to find 2 (and only 2) electrons. When a small volume centered around the nuclear position is used as starting guess, it is the $\Omega_{2}$ displayed on the left that is obtained after optimization. On the other hand, when a volume located far enough from the atomic position is used as the starting guess, the $\Omega_{2}$ solution displayed in the middle is obtained. The values of the maximum probabilities $p_{2}^{o p t}$, obtained at the end of the domain optimization, are displayed below the corresponding pictures. Since the code does not yet allow to automatically search for all different solutions, which is not a simple issue, it is the starting guess (both its form, size, and space localization), together with the maximization algorithm used, that determines which solution is found.

In this particular example, the $\Omega_{2}$ displayed on Fig. 1(a) can be associated to the core electron pair of the neon atom. A general feature of the probability function emanating from Eq. (2) is $p_{v}(\Omega)=p_{N-v}(\bar{\Omega})$. As a consequence, finding a domain $\Omega_{v}$ which maximizes the probability to find $v$ electrons, provides at the same time its complement, a domain maximizing the probability to find $N-v$ electrons $\left(\bar{\Omega}_{v}=\Omega_{N-v}\right)$. The complement of the domain $\Omega_{2}$ displayed in Fig. 1(a) thus corresponds to a domain maximizing the probability to find 8 electrons $\left(\Omega_{8}\right)$, which could be associated to the whole valence electron shell. The extremely high value of $p_{2}^{\text {opt }}$ indicates that there is a very strong structuration of electron pairs in atomic shells and very little fluctuation between shells. This has been found to be a general feature [14]. The domain obtained in Fig. 1(b) can be associated to one of the valence electron pairs, and due to the spherical symmetry of the atom any rotation of this domain around any axis passing through the atomic center would also provide a Maximum Probability Domain of $p_{2}$. Notice that the valence domains extend to infinity, so the external boundary found in the pictures is due to the maximization algorithm stopping at either a predetermined small $p_{2}$ gradient, at the grid limit, or as a consequence of applying an external pressure constraint. The maximal probability $p_{2}^{\text {opt }}$ obtained for this domain is much lower than the $p_{2}^{\text {opt }}$ corresponding to the atomic core pair, indicating that the valence electron pairs are much less structured than the cores.

This situation leads to two technical issues with domain optimization. Firstly, as core electron pairs correspond to very high maximal probabilities, they somehow act as "black holes" during the optimization process, and it is not uncommon when searching for a valence MPD that the core region is either found as a solution 
(if $v=2$ ), or included in the final MPD (if $v>2$ ). Secondly, core regions are small size volumes with a high electron density, a nearly spherical shape, and deforming them slightly strongly affects the probability, so that in order to be properly optimized very fine grids are necessary. Even though valence domains are searched, those valence domains which share a boundary with a core region (most of them) will also be affected by how finely the core region is represented, propagating the need of a fine mesh in their optimization. Since this leads to unnecessary large computing times when locating the chemically interesting valence MPDs, we have thus decided to get rid of the cores by using pseudopotentials in our calculations. The $\Omega_{2}$ valence MPD optimized for the neon atom using a VTZ basis including electron core pseudopotential is shown in the right part of Fig. 1(c). The MPD is very similar to the one obtained using an all-electron basis, except for the sharp shape near the nucleus as there is no core region now. The $p_{2}^{\text {opt }}$ value appears to be slightly higher to what has been obtained in the all-electron basis set, which is a common feature of pseudopotential vs. all-electron calculations, because in pseudopotential calculations the fluctuations between core and valence are eliminated.

Maximum Probability Domain optimizations have also been carried out for the halogen anions, which will be involved in the ionic bonds presented in the following. Table 1 displays the $p_{2}^{\text {opt }}$ values for the optimized valence $\Omega_{2}$ MPDs for the different atoms, along with the average population $\langle v\rangle$ defined as in Eq. (4) below.

$\langle v\rangle_{\Omega}=\sum_{v}^{N} v p_{v}$

The MPDs obtained are not reproduced here, as the solutions for the different halogen anions are both very similar one with the other, and with what is shown in Fig. 1(c). The $p_{2}^{\text {opt }}$ values are also all comparable, around 0.40 , which is a typical value for a well defined valence electron pair as will also be seen with the covalent and ionic bond domains. A last general remark is that although it is $p_{2}$ which is maximized during optimization, in the optimized MPD the average population $\langle v\rangle$ comes out close to 2 . According to our experience this is a quite general feature of MPDs: the average population in a $\Omega_{v}$ domain comes out close to $v$. The average population $\langle v\rangle$ is defined as the sum of the probabilities $p_{v}$ weighted by $v$, as shown in Eq. (4), and thus $\langle v\rangle$ being close to $v$ means that a close-to-perfect symmetric distribution of the probabilities around $p_{v}^{o p t}$ is obtained after optimization. There is no obvious physical reasons for that, in particular in dissymmetric molecules, as the only request during optimization is to maximize $p_{v}$. At present, we do not have an explanation to offer.

\subsection{Covalent bonding}

Fig. 2 displays two $\Omega_{2}$ MPDs corresponding to the two electron bond in ethane, and in trifluorimethyltrimethylsilane (TFMTMS) $\mathrm{F}_{3} \mathrm{CSiMe}_{3}$. This latter compound, best known as the RuppertPrakash reagent, is typically used in the addition of trifluoromethyl

Table 1

Optimized probability values $\left(p_{2}^{o p t}\right)$, and average electronic population $\left(\langle v\rangle\right.$ ) for the $\Omega_{2}$ MPD's corresponding to the valence domain for the Ne atom and the halogen anions.

\begin{tabular}{lll}
\hline & $p_{2}^{\text {opt }}$ & $\langle v\rangle$ \\
\hline $\mathrm{Ne}$ & 0.404 & 1.98 \\
$\mathrm{~F}^{-}$ & 0.403 & 1.98 \\
$\mathrm{Cl}^{-}$ & 0.400 & 1.98 \\
$\mathrm{Br}^{-}$ & 0.398 & 1.98 \\
$\mathrm{I}^{-}$ & 0.398 & 1.98 \\
\hline
\end{tabular}
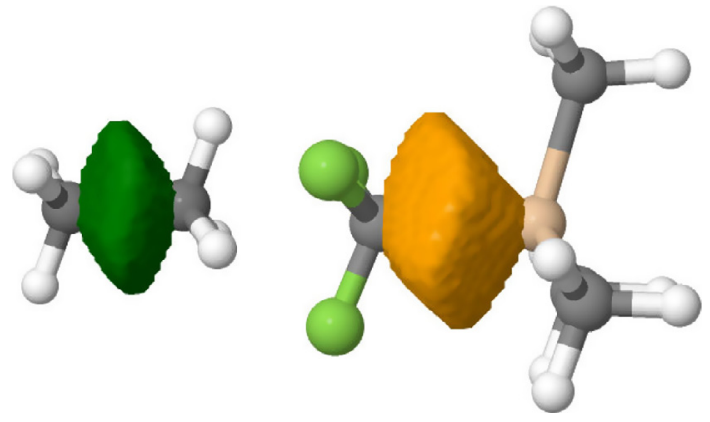
(a) $p_{2}^{o p t}=0.416$
(b) $p_{2}^{o p t}=0.488$

Fig. 2. $\Omega_{2}$ MPD's corresponding to the bonding region between the carbon atoms in ethane (figure (a)) and between the carbon and silicon atoms in TFMTMS, (figure (b)) together with their corresponding $p_{2}^{\text {opt }}$ probability values.

groups to organic compounds. For our purposes, this compound is expected to display a polar carbon-silicon two electron bond, as the electropositive character of the silicon atom is reinforced by the donating methyl substituents, whereas the more electronattractive character of the carbon atom is enhanced by the fluorine atoms. A Valence Bond BOVB calculation on both systems indeed provides a $25.8 \%$ weight for the major ionic structure in TFMTMS, $\mathrm{F}_{3} \mathrm{C}:{ }^{-}{ }^{+} \mathrm{SiMe}_{3}$, vs. a $11.9 \%$ weight for the minor ionic structure, $\mathrm{F}_{3} \mathrm{C}^{+-}: \mathrm{SiMe}_{3}$ whereas of course both ionic structures in ethane have equal weights ( $18.1 \%$ each).

The first important remark that can be made is that, contrarily to the usual experimental molecular chemists' view -inherited from the vision inferred from the modern version of Lewis' model, where a bond pair is depicted by an horizontal line connected two atoms - the spatial region in which the bonding electron pair has the largest probability does not extend along the bonding axis, but rather perpendicularly to the bond axis midpoint. This means that the chemically expected prolate spheroidal shape of a two electron bond should be substituted in the chemist mind by an oblate image, closer to what the MPD optimization shows.

A second significant remark is that, despite significant differences in polarity, the two bonding $\Omega_{2}$ regions for ethane and TFMTMS display a very similar shape, with no striking polarization of the electron pair towards the more electron-attractive fragment in a polar bond, as common chemical wisdom would indicate. It is however possible to better differentiate a polar bond like the carbon-silicon bond in TFMTMS from a non-polar bond like the carbon-carbon bond in ethane, by further dividing the $\Omega_{2}$ domains into two parts, each of them associated to one center. Although there are several ways to do this, for instance by using AIM atomic domains, we have chosen for the present, preliminary stage, to simply divide $\Omega_{2}$ by placing a vertical plane orthogonal to the carbon-carbon and carbon-silicon bond, such that the volumes on the left and right hand sides are roughly equal, and comparing $p_{2}$ in each part. For the ethane case, the probability in the two half-parts are of course identical, whereas they significantly differ in the case of TFMTMS, with a $p_{2 \text {,left }}=0.312$ in the half part closer to the more electron-attractive $\mathrm{CF}_{3}$ fragment, to be compared with a $p_{2, \text { right }}=0.121$ in the second half part. This indicates that the electron pair has a larger probability to be fully located close to $\mathrm{CF}_{3}$ fragment than close to the $\mathrm{SiMe}_{3}$ one. Note that the ratio $p_{2, \text { left }} / p_{2, \text { right }}$ is quite comparable to the ratio between the weights of the two ionic structures obtained with the BOVB method (25.8\%/11.9\%). We think that analyses in this kind of domains assigned to atoms might be a reliable way of assessing the polarity of a bond, compatible with the polarity obtained within Valence Bond theory. 
A question one may ask is whether other topological methods may also provide a faithful image of Lewis pairs. No such image comes out from the AIM analysis, where the space is partitioned into atomic regions. Besides, the basins of the Laplacian of the electron density do also encounter severe problems [36]. In the popular ELF method, we find disynaptic basins which are believed to provide an image of the bonding electron pair(s). In Fig. 3, the MPDs associated to the bonding pairs (top pictures) together with their corresponding ELF disynaptic basins (bottom pictures) are displayed for the ethane (left pictures) and dinitrogen (right pictures) molecules, respectively. The values of the target probabilities $\left(p_{v}\right)$, together with the average electron population $(\langle v\rangle)$ inside the regions, are also shown. In ethane, with a single carbon-carbon $\sigma$ bond, the relevant probability is $p_{2}$ and the expected average population is 2 , whereas for $\mathrm{N}_{2}$, displaying a triple bond, it is $p_{6}$ that should be compared to the (unique) disynaptic basin, with $p_{6}$ as the relevant probability and an expected average electronic population of 6 . In ethane the $\Omega_{2}$ MPD and the disynaptic basin are very similar. The $p_{2}$ probability inside the ELF basin is not far from the $p_{2}^{o p t}$ value found in the MPD, and the average population in the ELF basin is rather close to 2 . As a result, we can conclude that, for this molecule, the ELF disynaptic basin is a good approximation to the MPD associated to the bond. The situation is completely different for the $\mathrm{N}_{2}$ molecule, where the disynaptic basin is much smaller than the $\Omega_{6}$ MPD, together with an average population far away from the expected 6 electrons and a $p_{6}$ value close to 0 . In this latter case, the ELF disynaptic basin is not even a qualitatively acceptable approximation of the $\Omega_{6}$ MPD. The ELF basins have indeed a quite different physical meaning [37], and may or may not match the chemists' expectation of visualizing a volume that could be associated to the space-localization of the bonding electron pair(s). In the case of $\mathrm{N}_{2}$, in a paper to be found in this same special issue [38], one of us has indeed shown that the $\pi$ bonds in dinitrogen have substantially charge-shift character. It

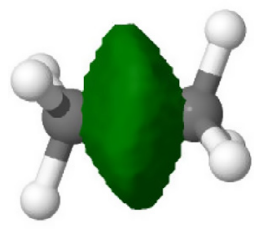

(a) $p_{2}=0.416,\langle\nu\rangle=1.98$

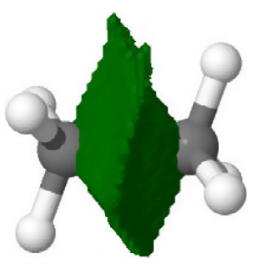

(c) $p_{2}=0.41,\langle\nu\rangle=1.88$

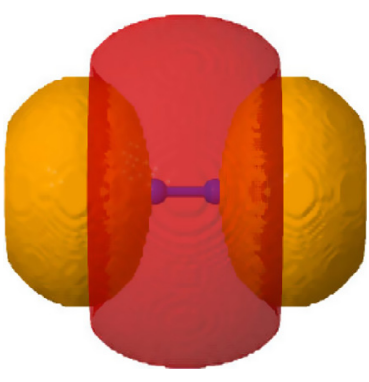

(b) $p_{6}=0.334,\langle\nu\rangle=6.00$

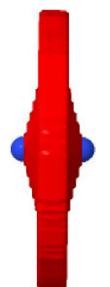

(d) $p_{6}=0.05,\langle\nu\rangle=3.64$
Fig. 3. Comparison between the appropriate MPD's (upper part) and ELF disynaptic basins (lower part) associated to the bonding region between $\mathrm{C}-\mathrm{C}$ in the ethane (left, $\Omega_{2}$ MPD depicted) and dinitrogen (right, $\Omega_{6}$ MPD) molecules, together with their relevant probability values $\left(p_{v}\right)$, and average electronic population $(\langle v\rangle)$. In (b), both the bonding $\mathrm{N}-\mathrm{N}$ domain (in red and made translucent) and the corresponding lone pairs region (in orange) are shown. Both of them were pressure constrained (see technical details). (For interpretation of the references to colour in this figure legend, the reader is referred to the web version of this article.) is known that charge-shift bonds lead to very small disynaptic basins, if any, and that a substantial part of the bonding density is found in these cases in the monosynaptic basins associated to the lone pairs [39].

\subsection{Ionic bonding}

Diatomic ionic bonded systems are usually viewed as made up of an atomic anion interacting with a cation through classical Coulomb forces. The most simple systems supposed to be quite ionic are $\mathrm{HX}$ molecules, composed of a hydrogen ion $\mathrm{H}^{+}$in interaction with a halogen anion $\mathrm{X}^{-}$(with $\mathrm{X}=\mathrm{F}, \mathrm{Cl}, \mathrm{Br}, \mathrm{I}$ ). As compared with the bare $\mathrm{X}^{-}$anions, which behave in a similar way as the Ne atom, the presence of a hydrogen ion nearby creates a symmetry lowering, so we do not expect to find anymore one sort of $\Omega_{2}$ symmetrically equivalent MPD in all spatial directions. The MPD search indeed provides two types of $\Omega_{2}$ solutions, displayed in Fig. 4 for the $\mathrm{HCl}$ molecule.

The first $\Omega_{2}$ shown is oriented along the $\mathrm{H}-\mathrm{Cl}$ axis and encloses the hydrogen atom. We thus associate it to the $\mathrm{H}-\mathrm{Cl}$ chemical bond. Note that, contrarily to what it is found for $\mathrm{C}-\mathrm{C}$ in ethane and for $\mathrm{C}-\mathrm{Si}$ in TFMTMS, in the particular case of the $\mathrm{H}-\mathrm{Cl}$ system this domain expands towards infinity along the left side of the internuclear axis (the domain in Fig. 4 was optimized without any pressure constraint), as there are no other electron pairs around the hydrogen atom that prevent such expansion. The second type of $\Omega_{2}$ solution obtained is directed towards the rear part of the $\mathrm{H}-\mathrm{X}$ region, one of such solutions being shown in Fig. 4(b), and might be associated to one of the halogen lone pairs. Because of the cylindrical symmetry, any rotation around the $\mathrm{H}-\mathrm{Cl}$ axis of one of such lone pair MPDs will also be a $\Omega_{2}$ solution. It is however possible to obtain by $\pm 120^{\circ}$ rotations of a first trial $\Omega_{2}$ lone pair two other $\Omega_{2}$ solutions. This set of three equivalent pairs are almost

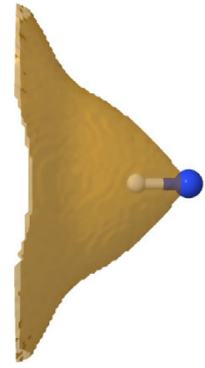

(a) $p_{2}^{o p t}=0.473$

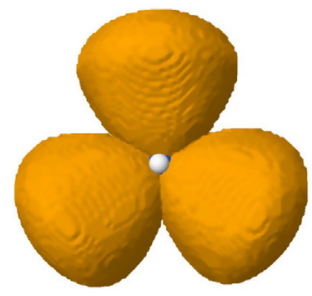

(c) Not a MPD

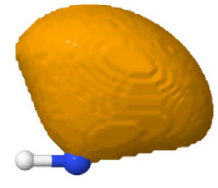

(b) $p_{2}^{o p t}=0.421$

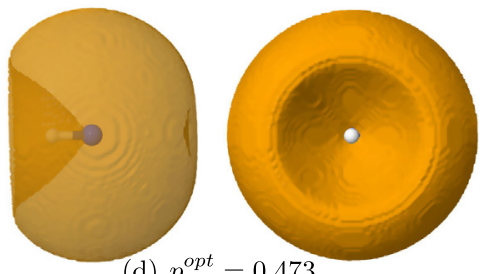

(d) $p_{6}^{o p t}=0.473$
Fig. 4. Different MPD's obtained for the $\mathrm{HCl}$ molecule (white: $\mathrm{H}$, blue: $\mathrm{Cl}$ ), together with their respective optimized probabilities $\left(p_{v}^{o p t}\right)$. Domain (a) is associated to the bonding region between hydrogen and chlorine atoms, whereas the domain (d) represents one of the halogen lone pair domains. Region (c) is the union of three $\Omega_{2}$ lone pair domains obtained by successive $120^{\circ}$ rotations of one of them (b) along the internuclear axis. The (d) MPD is obtained from the (c) region when $p_{6}$ is maximized. MPDs (b and d) have been optimized using an external pressure. (For interpretation of the references to colour in this figure legend, the reader is referred to the web version of this article.) 
disjoint with each one thus forming a representative image of the three lone pairs of the halogen atom, as shown in Fig. 4(c). Thus, the image of three VSEPR-like lone pairs around the $\mathrm{Cl}$ atom comes out quite naturally from an MPD analysis.

Actually, we think that considering these three pairs together captures more faithfully the situation in this system. To show it, we may look for a $\Omega_{6}$ domain by starting a $p_{6}$ optimization over the domain formed by the union of the three $p_{2}$ lone pairs just commented. The result shown in Fig. 4(d) is obtained. This $\Omega_{6}$ domain describes the three lone pairs altogether, and retrieves the cylindrical symmetry of the molecule as expected.

The global picture of the space repartition of the four valence electron pairs in $\mathrm{HCl}$ provided by the MPD method is thus compatible with the VSEPR rules, and can be summarized by Scheme 2, where the three pairs to the right of $\mathrm{X}$ may be collected together in a cylindrically symmetric lone pair region. The proximity between the images provided by the MPD solutions and the VSEPR model is not surprising, as the question: "maximize the probability to find 2 and only 2 electrons" can basically be considered as a probabilistic counterpart of the VSEPR criterion.

Similar solutions have also been obtained for all H-X systems, and are not displayed. In Table 2 we gather all data of interest concerning the optimized MPDs obtained for the different systems. Notice how similar the solutions are when going from one molecule to the other. The $\Omega_{2}$ associated with the $\mathrm{H}-\mathrm{X}$ bonds comes out with a $p_{2}^{\text {opt }}$ probability somehow larger than the one obtained for the $\Omega_{2}$ associated with the lone pairs, and with what has been obtained in the isolated halogen anions. This indicates that the lone pairs are less structured than the bond electron pair, with a larger fluctuation of lone pair electrons indicated by a larger variance (see Table 2), and thus that these three lone pairs are better understood if merged, eliminating the fluctuations between lone pairs. This is also illustrated by the higher $p_{6}^{\text {opt }}$ probability together with the smaller variance for the $\Omega_{6}$ MPD associated to the lone pairs. The $p_{2}^{o p t}$ value associated to the $\mathrm{H}-\mathrm{X}$ bonding domains slightly increases when going down in the periodic table for the halogen atom. This evolution is incidentally opposite in sense to that of the weight of the major ionic structure $\mathrm{H}^{+}{ }^{-}$: $\mathrm{X}$, which goes from $38.6 \%$ in the HF molecule to $26.4 \%$ in $\mathrm{HBr}$, i.e. $p_{2}^{\text {opt }}$ associated with this typical solution of polar-covalent bond logically rises with the covalency of the molecule. The $p_{2}^{\text {opt }}$ value for associated to the lone pairs is, in its turn, basically constant.

The vision depicted in Scheme 2 is not different from what has emerged for a covalent-polar bond as studied in Section 3.2, but one may wonder whether such is still the case in molecules displaying a larger polarity, where one of the ionic structure is expected to be the massively dominant.

The lithium halides $\mathrm{LiX}(\mathrm{X}=\mathrm{F}, \mathrm{Cl}, \mathrm{Br}, \mathrm{I})$ are expected to be such molecules. In these systems, a Valence Bond calculations on the LiF dimer provides a largely dominant $86.2 \%$ weight for the major ionic structure $\mathrm{Li}^{+-}: \mathrm{F}$, and still a $61.0 \%$ weight for the corresponding structure in Lil. Fig. 5 displays the MPD solutions which could be obtained for the $\mathrm{LiCl}$ molecule (the other halides show similar solutions). Now, and contrarily to what was found in all the previous systems, three different types of solutions have been obtained for the spatial arrangement of the bonding $v s$. the lone pairs. They are displayed in Fig. 5. The first solution, top, corresponds to the

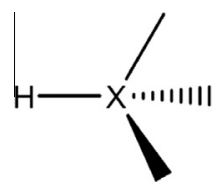

Scheme 2. Pairs arrangement found for the $\mathrm{HX}$ molecules ( $\mathrm{X}=\mathrm{F}, \mathrm{Cl}, \mathrm{Br}, \mathrm{I})$.
Table 2

Optimized probabilities ( $p_{v}^{\text {opt }}, v=2$ for $\Omega_{2}$ and $v=6$ for $\Omega_{6}$ ), population $(\langle v\rangle$ ), and variance $\left(\sigma^{2}\right)$ values for the bonding regions $\left(\Omega_{2}(\mathrm{a})\right)$ and for those domains corresponding to the lone pair over the halogen atom $\left(\Omega_{2}\right.$ (b)), as well as for the regions corresponding to the lone pairs $\left(\Omega_{6}(\mathrm{~d})\right)$ for each of the HX molecules (X $=\mathrm{F}$, $\mathrm{Cl}, \mathrm{Br}, \mathrm{I})$. Notice that $p_{6}\left(\Omega_{6}(\mathrm{~d})\right)$ has been optimized independently of $p_{2}\left(\Omega_{2}(\mathrm{a})\right)$, so that all the properties listed in the table except, of course, the average electronic population, should coincide for an exact optimization, the differences being due to numerical errors. The $\Omega_{2}$ (a) and $\Omega_{6}$ (b) MPDs have been subjected to pressure constraints. The domains' notation is identical to that given in Fig. 4.

\begin{tabular}{lllll}
\hline & & $p_{v}^{\text {opt }}$ & $\langle v\rangle$ & $\sigma^{2}$ \\
\hline$\Omega_{2}(\mathrm{a})$ & $\mathrm{HF}$ & 0.432 & 1.97 & 0.90 \\
& $\mathrm{HCl}$ & 0.473 & 1.97 & 0.78 \\
& $\mathrm{HBr}$ & 0.477 & 1.96 & 0.77 \\
& $\mathrm{HI}$ & 0.493 & 1.95 & 0.73 \\
$\Omega_{2}(\mathrm{~b})$ & $\mathrm{HF}$ & 0.412 & 1.98 & 0.95 \\
& $\mathrm{HCl}$ & 0.421 & 1.98 & 0.93 \\
& $\mathrm{HBr}$ & 0.420 & 1.98 & 0.93 \\
& $\mathrm{HI}$ & 0.423 & 1.98 & 0.92 \\
$\Omega_{6}(\mathrm{c})$ & $\mathrm{HF}$ & 0.432 & 6.03 & 0.90 \\
& $\mathrm{HCl}$ & 0.473 & 6.03 & 0.78 \\
& $\mathrm{HBr}$ & 0.476 & 6.04 & 0.77 \\
& $\mathrm{HI}$ & 0.493 & 6.05 & 0.73 \\
\hline
\end{tabular}
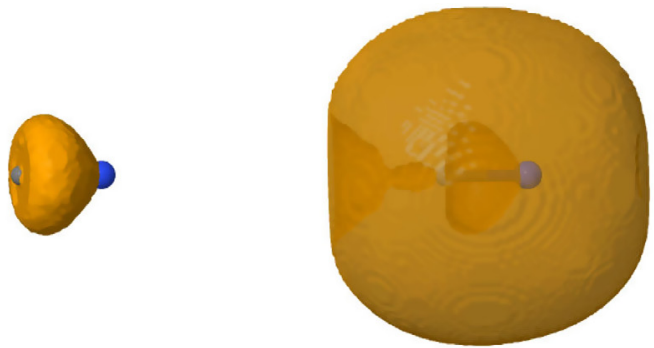

(a) $p_{2}^{o p t}=0.400$

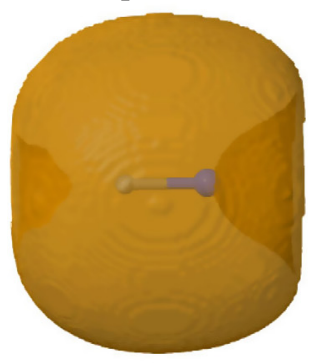

(c) $p_{6}^{o p t}=0.402$

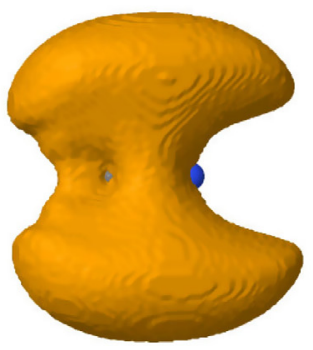

(e) $p_{4}^{o p t}=0.344$ (b) $p_{6}^{o p t}=0.400$

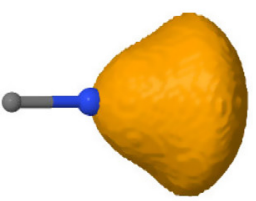

(d) $p_{2}^{o p t}=0.402$

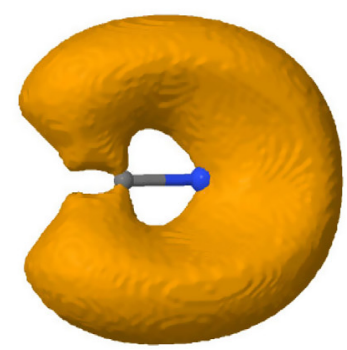

(f) $p_{4}^{\text {opt }}=0.344$
Fig. 5. Different MPD's obtained for the $\mathrm{LiCl}$ molecule (gray: $\mathrm{Li}$, blue: $\mathrm{Cl}$ ) together with their optimized probability values $\left(p_{v}^{o p t}\right)$. All the MPDs have been obtained with a pressure constraint. Domain (a) is associated to the bonding region between lithium and chlorine atoms, whereas domain (d) represents one of the halogen lone pair domains. Regions (b and c) are associated to two different conformations of three lone pair regions, being the complement of domains ( $a$ and d) respectively. Regions ( $\mathrm{e}$ and $\mathrm{f}$ ) are the MPDs consisting of the union of two lone pair domains. (For interpretation of the references to colour in this figure legend, the reader is referred to the web version of this article.) 
already known class of solutions that has been obtained for the polar-covalent bonds of the previous section and for the $\mathrm{H}-\mathrm{X}$ molecules, with a bonding $\Omega_{2}$ domain ((a)) located in between the two atoms, and a $\Omega_{6}$ domain being its space complement ((b)). However, this is not, in this case, the only solution. We have also been able to find the opposite type of pairs arrangement, shown in Fig. 5(d) and (c), with a $\Omega_{6}$ domain mainly localized between the two bonded atoms (left), and an axial lone pair oriented towards the rear part of the chlorine atom (right). In this arrangement, the lithium cation can be viewed as chelated by three pairs of electrons and electrostatically interacting with them. Last, but not least, a third intermediate arrangement has also been found, with two electron pairs of the chlorine pointing towards the central lithium ( $\Omega_{4}$ domain), and two other pairs oriented orthogonally to the previous ones located in opposition to the bonding region (Fig. 5(f) and (e)).

The global picture obtained for the pair arrangements in $\mathrm{LiCl}$ could be synthetized through Scheme 3. Hence, from this MPD analysis, bonding in systems having substantially ionic bonds could be viewed as a resonance between these three pair-arrangement situations. Note that it has been possible to retrieve multiple solutions in this case, because all these three solutions come out with comparable optimized probability, i.e. they are similarly probable, even if the arrangement in the two $\Omega_{4}$ domains comes
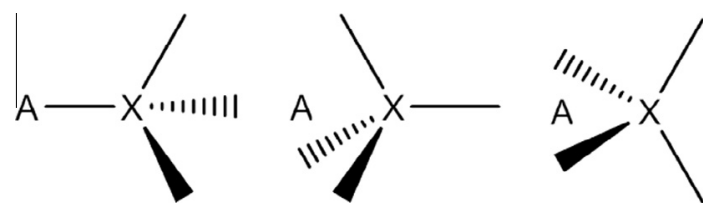

Scheme 3. Three different resonating structures for the pairs arrangements found in the $\operatorname{LiX}(\mathrm{X}=\mathrm{F}, \mathrm{Cl}, \mathrm{Br}, \mathrm{I})$ molecules.

\section{Table 3}

Optimized probabilities ( $p_{2}^{\text {opt }}$ for $\Omega_{2}, p_{4}^{\text {opt }}$ for $\Omega_{4}$ and $p_{6}^{\text {opt }}$ for $\Omega_{6}$ ), population ( $\langle v\rangle$ ), and variance $\left(\sigma^{2}\right)$ values for the bonding regions $\left(\Omega_{2}(\mathrm{a})\right)$ and for those domains corresponding to the lone pair over the halogen atom $\left(\Omega_{2}(\mathrm{~d})\right)$, as well as for the regions corresponding to the union of two lone pairs $\left(\Omega_{4}\right)$ and three lone pairs $\left(\Omega_{6}\right.$ (c)) for each of the LiX molecules (X $=\mathrm{F}, \mathrm{Cl}, \mathrm{Br}, \mathrm{I})$. Despite the fact that $\Omega_{2}(\mathrm{~d})$ and $\Omega_{6}$ (c) have been optimized independently, the probability values are identical, as well as the variance. The slightly differences in the last decimal digits are due to numerical errors. The domains' notation is identical to that given in Fig. 5.

\begin{tabular}{|c|c|c|c|c|}
\hline & & $p_{2}^{o p t}$ & $\langle v\rangle$ & $\sigma^{2}$ \\
\hline \multirow{4}{*}{$\Omega_{2}(\mathrm{a})$} & $\mathrm{LiF}$ & 0.399 & 1.98 & 1.00 \\
\hline & $\mathrm{LiCl}$ & 0.400 & 1.98 & 1.00 \\
\hline & $\mathrm{LiBr}$ & 0.400 & 1.98 & 1.01 \\
\hline & LiI & 0.398 & 1.99 & 1.01 \\
\hline \multirow[t]{5}{*}{$\Omega_{2}(\mathrm{~d})$} & $\mathrm{LiF}$ & 0.408 & 1.98 & 0.97 \\
\hline & $\mathrm{LiCl}$ & 0.402 & 1.98 & 0.99 \\
\hline & $\mathrm{LiBr}$ & 0.398 & 1.98 & 1.01 \\
\hline & LiI & 0.399 & 1.98 & 1.01 \\
\hline & & $p_{4}^{o p t}$ & $\langle v\rangle$ & $\sigma^{2}$ \\
\hline \multirow[t]{4}{*}{$\Omega_{4}(\mathrm{f})$} & $\mathrm{LiF}$ & 0.346 & 4.00 & 1.32 \\
\hline & $\mathrm{LiCl}$ & 0.344 & 4.00 & 1.34 \\
\hline & $\mathrm{LiBr}$ & 0.342 & 4.00 & 1.35 \\
\hline & LiI & 0.341 & 4.00 & 1.37 \\
\hline \multirow[t]{5}{*}{$\Omega_{4}(\mathrm{e})$} & $\mathrm{LiF}$ & 0.346 & 4.00 & 1.32 \\
\hline & $\mathrm{LiCl}$ & 0.344 & 4.00 & 1.34 \\
\hline & $\mathrm{LiBr}$ & 0.342 & 4.00 & 1.35 \\
\hline & LiI & 0.341 & 4.00 & 1.37 \\
\hline & & $p_{6}^{o p t}$ & $\langle v\rangle$ & $\sigma^{2}$ \\
\hline \multirow[t]{4}{*}{$\Omega_{6}(\mathrm{c})$} & $\mathrm{LiF}$ & 0.408 & 6.02 & 0.97 \\
\hline & $\mathrm{LiCl}$ & 0.402 & 6.02 & 0.99 \\
\hline & $\mathrm{LiBr}$ & 0.398 & 6.02 & 1.01 \\
\hline & LiI & 0.398 & 6.02 & 1.01 \\
\hline
\end{tabular}

out with a somehow lower probability than the two others. This situation is reminiscent of the infinitely equivalent solutions of pair arrangement found in the bare neon atom and halogen ions due to the spherical symmetry. Now in LiCl the spherical symmetry is lost but, nevertheless, this almost free rotation of the electron pairs around the $\mathrm{Cl}^{-}$ion persists somehow. These results provide a unique vision of the ionic bond, as a lithium cation interacting with all the four free rotating electron pairs of the corresponding chlorine atom in three types of resonating structures.

This interesting image appears to be quite general in the different ionic LiX bonds, as such resonating solutions come out for all the molecules of the LiX series. The shapes of these MPDs (not shown) are similar to those displayed in $\mathrm{LiCl}$, with only marginal quantitative variation among systems. The values of the probabilities are also quite comparable, as shown in Table 3.

\section{Conclusions}

Maximum Probability Domains appear as a promising way of analysing the electronic structure of molecules in real space. Both the definition of the probability function and the physical meaning of the domains are conceptually and mathematically simple, and can be understood with only basic knowledge of Quantum Mechanics. MPDs readily provide pictures that can be associated to the arrangement of electrons or electron pairs in real space, they can be extracted, in principle, from any type of wave function, and thus may be used to establish a direct connection between accurate Quantum Mechanical calculations and the chemists' Lewis-like vision. From analyses on bare atoms a close to perfect separation of electrons in terms of shells has been found, confirming previous studies. The MPD vision of the two-electron polarcovalent bond is particularly interesting, with prolate spheroidal domains at variance with commonly seen oblate images around the bond axis. More polar bonds display only slightly dissymmetric domains, although polarity mith still be identified, and possibly quantified, through probability analyses carried out by dividing MPDs into atomic-like domains. In this contribution we have shown how this can be done by using equal volume half-domains, and we have proposed to use AIM domains in future applications. From the MPD analysis on dominantly ionic bonds, namely the LiX $(\mathrm{X}=\mathrm{F}, \mathrm{Cl}, \mathrm{Br}, \mathrm{I})$ systems, an appealing resonating picture of a lithium cation interacting with three types of dynamically changing space-arrangements of the halogen anion electron pairs has emerged. These different pair arrangements are all almost equiprobable in these bonds.

\section{Acknowledgments}

M.M. and A.M.P. are grateful to the spanish government for financial support, Grants BES-2010-032781 and CTQ-2012-31174.

The authors thank Osvaldo Mafra Lopes Jr. and Prof. Wei Wu, for having made their codes available to us.

\section{References}

[1] G.N. Lewis, The atom and the molecule, J. Am. Chem. Soc. 38 (1916) 762-785.

[2] I. Langmuir, The arrangement of electrons in atoms and molecules, J. Am. Chem. Soc. 41 (1919) 868-934.

[3] L. Pauling, The nature of the chemical bond. Application of results obtained from the Quantum Mechanics and from a theory of paramagnetic susceptibility to the structure of molecules, J. Am. Chem. Soc 53 (1931) 1367-1400.

[4] (a) G. Sini, P. Matre, P.C. Hiberty, S.S. Shaik, Covalent, ionic and resonating single bonds, J. Mol. Struct. (THEOCHEM) 229 (1991) 163-188;

(b) S.S. Shaik, P. Matre, G. Sini, P.C. Hiberty, The charge-shift bonding concept. Electron-pair bonds with very large ionic-covalent resonance energies, J. Am. Chem. Soc. 114 (1992) 7861-7866;

(c) S. Shaik, D. Danovich, W. Wu, P.C. Hiberty, Charge-shift bonding and its manifestations in chemistry, Nat. Chem. 1 (2009) 443-449. 
[5] W. Wu, P. Su, S. Shaik, P.C. Hiberty, Classical Valence Bond approach by modern methods, Chem. Rev. 111 (2011) 7557-7593.

[6] B. Braïda, E. Derat, S. Humbel, P.C. Hiberty, S. Shaik, The Valence Bond workshop in Paris: the phoenix rises from the ashes or, has a love story with MO-based theories begun?, Chem Phys. Chem. 13 (2012) 4029-4030.

[7] D. Usharani, W. Lai, C. Li, H. Chen, D. Danovich, S. Shaik, A tutorial for understanding chemical reactivity through the Valence Bond approach, Chem. Soc. Rev. 43 (2014) 4968-4988.

[8] (a) Z. Chen, Q. Zhang, W. Wu, A new algorithm for energy gradients and orbital optimization in Valence Bond theory, Sci. China B 39 (2009) 1424-1429; (b) X. Chen, Q. Cheng, J. Song, X. Chi, W. Wu, Using automatic differentiation in the optimization of Xiamen software, J. Numer. Meth. Comput. Appl. 30 (2009) 21-29;

(c) J. Song, Z. Chen, S. Shaik, W. Wu, An efficient algorithm for complete active space Valence Bond self-consistent field calculation, J. Comp. Chem. 34 (2013) 38-48;

(d) Z. Chen, X. Chen, W. Wu, Nonorthogonal orbital based N-body reduced density matrices and their applications to valence bond theory. II. An efficient algorithm for matrix elements and analytical energy gradients in VBSCF method, J. Chem. Phys. 138 (2013) 164120:1-164120:12.

[9] R.F.W. Bader, Atoms in Molecules, Oxford University Press, Oxford, 1990.

[10] A.D. Becke, K.E. Edgecombe, A simple measure of electron localization in atomic and molecular systems, J. Chem. Phys. 92 (1990) 5397-5403.

[11] B. Silvi, A. Savin, Classification of chemical bonds based on topological analysis of electron localization functions, Nature 371 (1994) 683-686.

[12] E.R. Johnson, S. Keinan, P. Mori-Sánchez, J. Contreras-García, A.J. Cohen, W. Yang, Revealing noncovalent interactions, J. Am. Chem. Soc. 132 (2010) 64986506.

[13] J. Contreras-García, E.R. Johnson, S. Keinan, R. Chaudret, J.P. Piquemal, D.N Beratan, W. Yang, NCIPLOT: a program for plotting noncovalent interaction regions, J. Chem. Theo. Comp. 7 (2011) 625-632.

[14] A. Savin, in: K.D. Sen (Ed.), Reviews of Modern Quantum Chemistry, World Scientific Publishing, Singapore, 2002.

[15] E. Chamorro, P. Fuentealba, A. Savin, Electron probability distribution in AIM and ELF basins, J. Comput. Chem. 24 (2003) 496-504.

[16] E. Francisco, A. Martín Pendás, M.A. Blanco, Electron number probability distributions for correlated wave functions, J. Chem. Phys. 126 (2007) 094102:1-13.

[17] A. Martín Pendás, E. Francisco, M.A. Blanco, Spin resolved electron number distribution functions: how spins couple in real space, J. Chem. Phys. 127 (2007). 144103:1-10.

[18] E. Francisco, A. Martín Pendás, M.A. Blanco, EDF: computing electron number probability distribution functions in real space from molecular wave functions, Comp. Phys. Commun. 178 (2008) 621-634.

[19] A. Gallegos, R. Carbó-Dorca, F. Lodier, E. Cancès, A. Savin, Maximal probability domains in linear molecules, J. Comput. Chem. 26 (2005) 455-460.

[20] A. Scemama, M. Caffarel, A. Savin, Maximum probability domains from Quantum Monte Carlo calculations, J. Comput. Chem. 28 (2007) 442-454.

[21] O. Mafra Lopes Jr., B. Braïda, M. Causà, A. Savin, Understanding maximum probability domains with simple models, Prog. Theor. Chem. Phys. 22 (2011) 175-186.

[22] M. Menéndez, A. Martín Pendás, On the stability of some analytically solvable Maximum Probability Domains, Theor. Chem. Acc. 133 (2014) 1539:1-8.

[23] (a) M. Causà, A. Savin, Maximum probability domains in the solid-state structures of the elements: the diamond structure, Z. Anorg. Allg. Chem 637 (2011) 882-884;

(b) M. Causà, A. Savin, Maximum probability domains in crystals: the rock-salt structure, J. Phys. Chem. A 115 (2011) 13139-13148;

(c) M. Causà, M. D’Amore, C. Garzillo, et al., Applications of Density Functional Theory to biological and bioinorganic Chemistry, in: M.V. Putz, D.M. Mingos (Eds.), Book Series: Strucure and Bonding, vol. 150, Springer, Berlin, Heidelberg, 2013, pp. 119-141.
[24] Gaussian 03, Revision C.02, M.J. Frisch, G.W. Trucks, H.B. Schlegel, G.E. Scuseria, M.A. Robb, J.R. Cheeseman, J.A. Montgomery, Jr., T. Vreven, K.N. Kudin, J.C. Burant, J.M. Millam, S.S. Iyengar, J. Tomasi, V. Barone, B. Mennucci, M. Cossi, G. Scalmani, N. Rega, G.A. Petersson, H. Nakatsuji, M. Hada, M. Ehara, K. Toyota, R. Fukuda, J. Hasegawa, M. Ishida, T. Nakajima, Y. Honda, O. Kitao, H. Nakai, M. Klene, X. Li, J.E. Knox, H.P. Hratchian, J.B. Cross, C. Adamo, J. Jaramillo, R. Gomperts, R.E. Stratmann, O. Yazyev, A.J. Austin, R. Cammi, C. Pomelli, J.W. Ochterski, P.Y. Ayala, K. Morokuma, G.A. Voth, P. Salvador, J.J. Dannenberg, V.G. Zakrzewski, S. Dapprich, A.D. Daniels, M.C. Strain, O. Farkas, D.K. Malick, A.D. Rabuck, K. Raghavachari, J.B. Foresman, J.V. Ortiz, Q. Cui, A.G. Baboul, S. Clifford, J. Cioslowski, B.B. Stefanov, G. Liu, A. Liashenko, P. Piskorz, I. Komaromi, R.L. Martin, D.J. Fox, T. Keith, M.A. Al-Laham, C.Y. Peng, A Nanayakkara, M. Challacombe, P.M.W. Gill, B. Johnson, W. Chen, M.W. Wong, C. Gonzalez, and J.A. Pople, Gaussian, Inc., Wallingford CT, 2004.

[25] (a) M. Burkatzki, C. Filippi, M. Dolg, Energy-consistent pseudopotentials for QMC calculations, J. Chem. Phys. 126 (2007) 234105:1-234105:8; (b) http://www.burkatzki.com/pseudos/index.2.html.

[26] The improved pseudopotential for hydrogen atom was taken from the Supplementary Material of the following article: F.R. Petruzielo, J. Toulouse, C.J. Umrigar, Approaching chemical accuracy with quantum Monte Carlo, J. Chem. Phys. 136 (2012) 124116:1-5.

[27] T.H. Dunning Jr., Gaussian basis sets for use in correlated molecular calculations. I. The atoms boron through neon and hydrogen, J. Chem. Phys. 90 (1989) 1007-1023.

[28] O. Mafra Lopes Jr., Retrouver les structures de Lewis partir des fonctions d'onde, PhD thesis, École Doctorale de Chimie Physique et Chimie Analytique de Paris-Centre, 2010.

[29] S. Noury, X. Krokidis, F. Fuster, B. Silvi, Computational tools for the electron localization function topological analysis, Comput. Chem. 23 (1999) 597-604.

[30] L. Song, Y. Mo, Q. Zhang, W. Wu, XMVB: a program for ab initio nonorthogonal valence bond computations, J. Comput. Chem 26 (2005) 514-521.

[31] M.W. Schmidt, K.K. Baldridge, J.A. Boatz, S.T. Elbert, M.S. Gordon, J.H. Jensen, S. Koseki, N. Matsunaga, K.A. Nguyen, S. Su, T.L. Windus, M. Dupuis, J.A Montgomery, General atomic and molecular electronic structure system, J. Comput. Chem. 14 (1993) 1347-1363.

[32] (a) P.C. Hiberty, J.P. Flament, E. Noizet, Compact and accurate valence bond functions with different orbitals for different configurations: application to the two-configuration description of $F_{2}$, Chem. Phys. Lett. 189 (1992) 259-265; (b) P.C. Hiberty, S. Humbel, C.P. Byrman, J.H. van Lenthe, Compact valence bond functions with breathing orbitals: application to the bond dissociation energies of $F_{2}$ and FH, J. Chem. Phys. 101 (1994) 5969-5976; (c) P.C. Hiberty, S. Shaik, Breathing-orbital valence bond method - a modern valence bond method that includes dynamic correlation, Theor. Chem. Acc. 108 (2002) 255-272.

[33] H.B. Chirgwin, C.A. Coulson, The electronic structure of conjugated systems. VI, Proc. Roy. Soc. London A 201 (1950) 196-209.

[34] Jmol: an open-source Java viewer for chemical structures in 3D. http:// www.jmol.org/.

[35] E. Cancès, R. Keriven, F. Lodier, A. Savin, How electrons guard the space: shape optimization with probability distribution criteria, Theor. Chem. Acc. 111 (2004) 373-380.

[36] A. Otero de la Roza, V. Luaña, Topological characterization of the electron density Laplacian in crystals, The case of the group IV elements, J. Chem. Theory Comput. 6 (2010) 3761-3779.

[37] A. Savin, On the significance of the ELF basins, J. Chem. Sci. 117 (2005) 473-475.

[38] K. Hendrickx, B. Braïda, P. Bultinck, P.C. Hiberty, More insight in multiple bonding with Valence Bond theory, Comp. Theor. Chem. (2014). "in this Special Issue".

[39] S. Shaik, D. Danovich, B. Silvi, D.L. Lauvergnat, P.C. Hiberty, Charge-shift bonding - a class of electron-pair bonds that emerges from valence bond theory and is supported by the electron localization function approach, Chem. A Eur. J. 11 (2005) 6358-6371. 